Burrows is an eminent and productive figure in neurobiology, and this book is an immensely authoritative treatment of locust neurobiology. His message is clear and important: while it is true that neurobiology has made huge progress, it is critical not to lose sight of the fundamental importance of detailed, experimental analyses of real nervous systems. It is because of such studies that we are where we are today. There is still much to learn, and the danger is that fashion will drive neurobiology to the point where computational and molecular approaches find themselves uncoupled from the functioning nervous system.

Burrows has chosen to concentrate on those areas of locust neurobiology where he has had greatest impact. A brief introductory chapter on locust biology is followed by chapters on the anatomy, components and development of the nervous system. He then proceeds to consider chemical communication within the nervous system before concentrating on the control of local leg movements, walking, jumping, escape, flying and breathing. Where they provide relevant data, other insects are made honorary locusts. The treatment is detailed, critical and well illustrated. Burrows points out where dogmas are unsubstantiated (one example he gives was based on a single published trace). He offers detailed assessments of the state of current knowledge, highlights lacunae and suggests areas for future research. The result is a key reference work, which should be read by all neurobiologists and behaviourists. After all, we still do not fully understand how a locust moves its leg away from a touch, let alone how a cockroach escapes.

Stephen J. Simpson is in the Department of Zoology, University of Oxford, South Parks Road, Oxford OXI 3PS.

\section{Infinite possibilities}

\section{Before the Beginning: Our Universe and Others \\ by Martin Rees \\ Simon \& Schuster: 1997. Pp. 282. $£ 16.99$ \\ Joseph Silk}

Before the Beginning is an unusual blend of wit, asperity and cosmology, the last based around an anthropic core. The Astronomer Royal, Sir Martin Rees, takes the reader on an enthralling tour of the 'multiverse', defined by him as the ensemble of all possible universes, which he confidently asserts to be part of cosmological reality. Yet this metaphysical agglomeration of universes is inherently unobservable, because all but one, our own delicately balanced cosmos, is inimical to intelligent life.

The many-universe postulate is intellectually challenging, and purports to explain a plethora of unlikely circumstances. Why

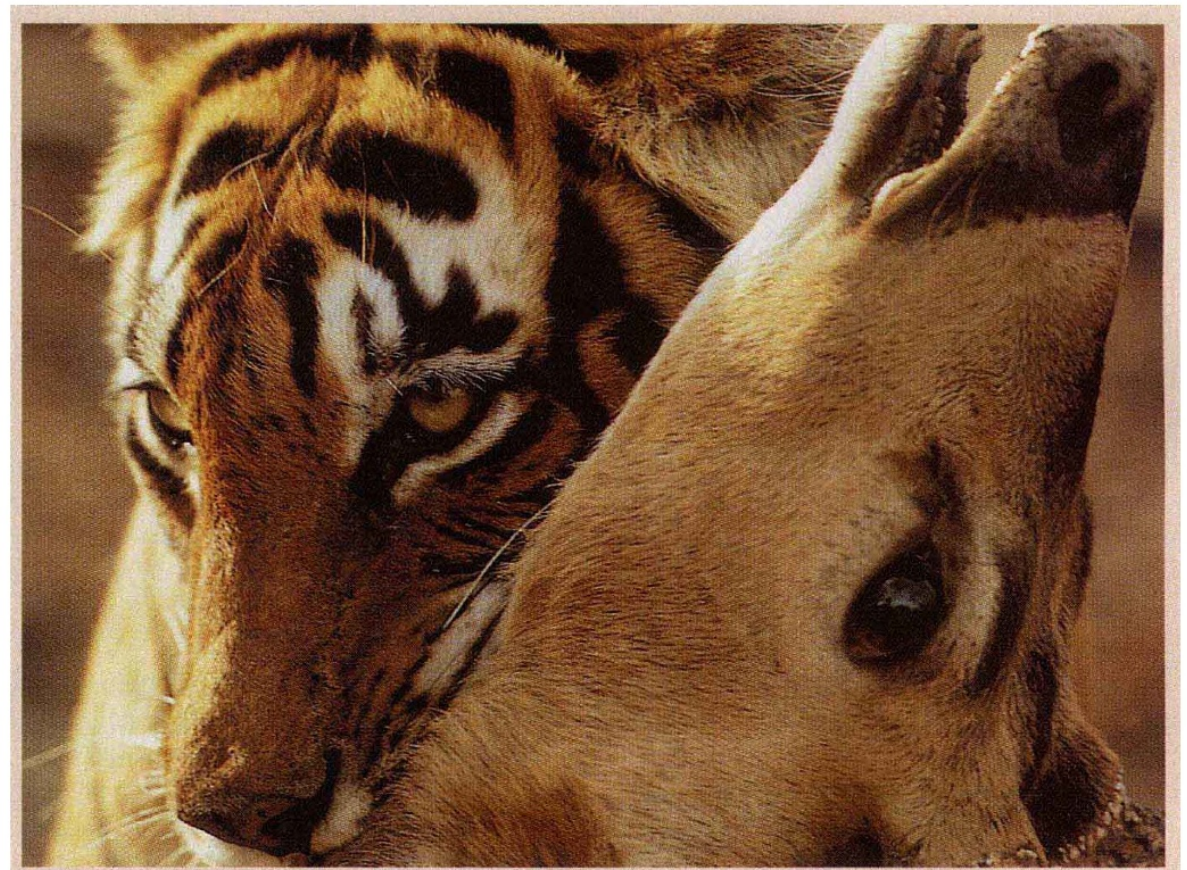

\title{
A matter of life and death
}

India's tiger population, the largest in the world, is under threat from poaching. The story of a family of these magnificent animals is told in the

does the essence of life, the carbon atom, have an energy level in just the right place to enable its constituent helium atoms to be captured before they fly apart in stellar cores? Why is the vacuum empty, or almost so? Why is the nuclear force just the right strength to enable stars to form? Why is the 'weak force' as weak as it is, enabling elements to form? Why is the neutron just 14 per cent more massive than the proton, enabling hydrogen to form? Why is the electron mass only $1 / 1,836$ of the proton mass (were it much larger, molecules such as DNA would not form)? Why were density fluctuations in the early Universe small enough, but not too small, for galaxies to form? The list seems endless.

How easy it is to postulate an infinity of universes that violate these precepts, and thereby remain unobserved, devoid of cosmologists and their books. Surely there must be some underlying theory that provides a physical explanation? Rees takes a stab at one when he eloquently describes black holes. Embedded within black holes may be other universes. A worm hole, which connects a black hole to its opposite, a white hole, would be the port of entry to a new universe that may be newly formed or ancient, and remote (or possibly not) in space and time from its parent universe. There are untold numbers of black holes in the universe. Perhaps there is an infinity of universes within universes. These may provide the anthropic framework that could explain practically all unsolved mysteries of the cosmos.

The average mind spins at this concept, lavishly illustrated A Tiger's Tale: The Indian Tiger's Struggle for Survival in the Wild by Anup and Manoj Shah (Fountain Press, £24.95).

but cosmologists dauntlessly explore the consequences. The most bizarre concept is that of evolution of the baby universes: spin-off universes could display the effects of heredity and environment. Optimal procreation would require a furious proliferation of black holes, a prediction conceivably testable by X-ray astronomers.

Despite these speculative themes which permeate the book, Rees classifies himself as a reluctant conservative in his approach to science. I found myself wondering what views a radical cosmologist might espouse. Probably such a person would have included illustrations. Certainly that would lighten the text, although the author's descriptions of technical issues are noteworthy for their unusual combination of clarity and conciseness. Rees displays his political astuteness: he assesses the satellite experiment Gravity Probe-B and the cost of the Space Telescope, without seemingly taking sides. He is clearly a gambler at heart: I counted at least five bets on burning cosmological issues.

Perhaps laying odds is a subtle way of sidestepping the embarrassingly naive tendency, displayed by otherwise respectable colleagues in recent popular tracts on cosmology, to invoke theological issues. Rees is to be commended for telling the unadorned story of the latest developments in cosmology in a forthright and compelling manner.

Joseph Silk is at the Institut d'Astrophysique, 98 bis, Boulevard Arago, F-75014 Paris, France, and is on leave from the University of California at Berkeley, California 94720, USA. 